\title{
El comité comunitario en el manejo de recursos hidrológicos en San José Chiltepec, Oaxaca, México
}

\author{
The community committee on hydrological resources management \\ in San José Chiltepec, Oaxaca, Mexico \\ Marco Aurelio Acevedo-Ortiz ${ }^{*}$, Yolanda Donaji Ortiz-Hernández ${ }^{1}$, Rafael Pérez-Pacheco ${ }^{1}$, \\ Gema Lugo-Espinosa²
}

\begin{abstract}
RESUMEN
El objetivo de este trabajo es analizar el proceso del establecimiento de un comité comunitario y su involucramiento para mejorar las condiciones ambientales bajo una lógica de acción colectiva en San José Chiltepec, Oaxaca, México; donde existe un problema de abastecimiento de agua potable y contaminación en aguas superficiales. Para este fin, se involucró a la comunidad en la mejora de su situación ambiental mediante talleres participativos. Los principales resultados muestran que la acción colectiva voluntaria de conservación ha contribuido el establecimiento de un comité local, derivando de ello, el diseño de un reglamento interno para normar acciones orientadas al saneamiento de las fuentes de agua; y la validación del documento ante la asamblea comunitaria. El comité se presenta como un caso de éxito por el grado de participación local, pero requiere fortalecer capacidades y un acompañamiento institucional para lograr la continuidad y escalamiento a nivel regional.
\end{abstract}

Palabras clave: Autogestión, recursos naturales; empoderamiento, reglamentos internos, comunidades rurales.

\section{ABSTRACT}

The objective of this work is to analyze the process of establishing a community committee and its involvement to improve environmental conditions under a logic of collective action in San José Chiltepec, Oaxaca, Mexico; where a problem of supply of drinking water and contamination in surface waters exists. To this end, the community was involved in improving its environmental situation through participatory workshops. The main results show that voluntary collective conservation action has contributed to the establishment of a local committee, deriving from this, the design of an internal decree to regulate actions aimed at the sanitation of water sources; and the validation of the document before the community assembly. The committee is presented as a success story due to the degree of local participation, but it requires strengthening capacities and institutional support to achieve continuity and scaling at the regional level.

Keywords: self-management, natural resources; Empowerment, internal regulations, rural communities.

\section{Introducción}

El hombre siempre ha buscado fuentes de alimento, agua en cantidad suficiente y con calidad adecuada para sobrevivir (Ballesteros, et al., 2010). En México, los recursos hidrológicos (446,777 $\mathrm{hm}^{3} /$ anuales) son bienes promotores del desarrollo sustentable (CONAGUA, 2016) y su disponibilidad está sujeta a un modelo de explotación que destina el 77\% del total extraído a la agricultura y ganadería (OCDE, 2013).
Día con día, los recursos naturales son más limitados, volviéndose necesario replantear la forma de atender los problemas de abastecimiento de agua y revalorar este elemento vital debido a su escasez. En México existen 653 acuíferos de aguas subterráneas a nivel nacional que aportan el $38.9 \%$ del agua requerida por los diferentes sectores; 105 se encuentran en estado de sobreexplotación, 32 con presencia de suelos salinos y agua salobre y 18 con intrusión marina (CONAGUA, 2016). De los 1,449,471 millones de $\mathrm{m}^{3}$ de precipitación que recibe

\footnotetext{
1 Instituto Politécnico Nacional. CIIDIR-Oaxaca. Santa Cruz Xoxotlán, Oaxaca, México.

2 Fondo Ambiental Regional de la Chinantla, Oaxaca. A.C., Tuxtepec, Oaxaca, México.

* Autor para correspondencia: yortiz@ipn.mx
}

Fecha de Recepción: 23 Junio, 2017.

Fecha de Aceptación: 12 Octubre, 2017.

DOI: 
México, sólo el $21.2 \%$ escurre por ríos y arroyos y el $6.3 \%$ se puede infiltrar al subsuelo de forma natural y recarga los acuíferos (CONAGUA, 2016).

México implementó el modelo de cuencas hidrológicas en 1989 mediante la Comisión Nacional del Agua y publicó un marco jurídico en 1992 para regular el agua y su uso de forma integral. Se incluyeron modificaciones a la Ley de Aguas Nacionales, dando origen a los Organismos Operadores de Agua Potable (OOAP), con la facultad de concesionar el manejo administrativo a una empresa privada (Carrasco y Vargas, 2012).

A su vez, se gestaron apoyos a través del Programa de Modernización de Organismos Operadores de Agua (PROMAGUA) financiado por el Banco Interamericano de Desarrollo mediante apoyos orientados a incentivar la creación de nuevos OOAP y una tendencia clara a la privatización de los recursos naturales estratégicos (Carrasco y Vargas, 2012). La gestión del agua en los centros urbanos/ rurales no sólo debe de tener una visión de manejo administrativa, sino también humanista (Dourejani, 1999), en donde el agua para consumo humano sea prioritaria dentro de las políticas públicas. Por tanto, no existían lineamientos que vincularan a los OOAP con las comunidades/ciudades sobre la responsabilidad social que debieran cumplir al administrar los recursos de la nación.

El gobierno mexicano en el Programa Nacional Hídrico 2014-2018 reconoce cambios en la forma de percibir el agua (inagotable) hacia un modelo más sustentable. No obstante, la eficiencia en el uso del agua continuaba siendo muy baja y su demanda constante (OCDE, 2013), sin obviar los problemas que aún continúan vigentes: a) conflictos territoriales; b) falta de involucramiento de actores locales; c) normatividad escasa para operatividad de organismos operadores; y d) capacidades instaladas (Perevochtchikova y Arellano-Monterrosas, 2008). Situación muy similar a la presentada en las áreas de conservación decretadas por gobiernos (DeFries et al., 2005; Liu et al., 2010) al forzar un manejo sustentable y/o de conservación exclusivo.

Sin embargo, existe poca investigación sobre los sistemas comunitarios de agua en México y es poco documentada (Galindo y Palerm, 2016). La mayoría de las investigaciones que se realizan en el país, se basa en el análisis a organismos locales (rurales o urbanos) ya existentes, o de otros países como lo es el caso de los Organizaciones Comunitarias proveedoras de Aguas y Saneamiento
(Ochoa, et al., 2011) que han surgido como una alternativa para la gestión rural del agua.

Por tanto, el objetivo que se muestra en el presente caso de estudio fue el análisis del proceso de establecimiento de un comité comunitario para mejorar las condiciones ambientales bajo una lógica de acción colectiva en el municipio de San José Chiltepec, donde existen problemas de contaminación en aguas superficiales; y su involucramiento en la generación de un marco normativo a nivel ejidal complementario a su reglamento interno como detonante de acciones para el manejo de recursos hidrológicos.

\section{Materiales Y Métodos}

\section{Ubicación y zona de estudio}

Oaxaca cuenta con 570 (25\%) municipios de los 2,438 que existen en México, y se divide en ocho regiones. La región del Papaloapan - Chinantla, se extiende a lo largo de 14 municipios y 450 localidades. Con una superficie de 282,877.86 ha de selva alta perennifolia, 62,023.87 ha de Bosque Mesófilo de Montaña y 19,800 ha de Bosque de Pino/Encino, contribuye a que el agua renovable disponible en la región sureste de México sea siete veces mayor al resto de regiones hidrológico-administrativas (CONAGUA, 2016). No obstante, en las zonas rurales de Oaxaca, el sistema de agua potable apenas tiene una cobertura del $40 \%$ y el de alcantarillado del $5 \%$, mientras que las ca nivel nacional la media es del $64 \%$ y $25 \%$, respectivamente.

El estado de la cuenca del río Papaloapan, en el año 2000 (CONAGUA, 2016) era considerado un $70 \%$ contaminado, con un promedio de 197,000 ton/año de material contaminante de Demanda Bioquímica de Oxígeno (DBO5), proveniente de industrias $(89 \%)$, municipios $(10 \%)$ y otros sectores $(1 \%)$.

Como zona de estudio se eligió el municipio de San José Chiltepec, que forma parte de las Zonas de Atención Prioritaria Urbana y de la cuenca del río Papaloapan; posee 5,787 has de selva alta perennifolia; 4,488 has de pastizales; 1,064 has de vegetación secundaria y 87 has de zonas urbanas; y por su territorio cruza el río Valle Nacional (Figura 1).

En el municipio existen 2,638 viviendas, de las cuáles: $43.7 \%$ no tiene acceso al sistema de 

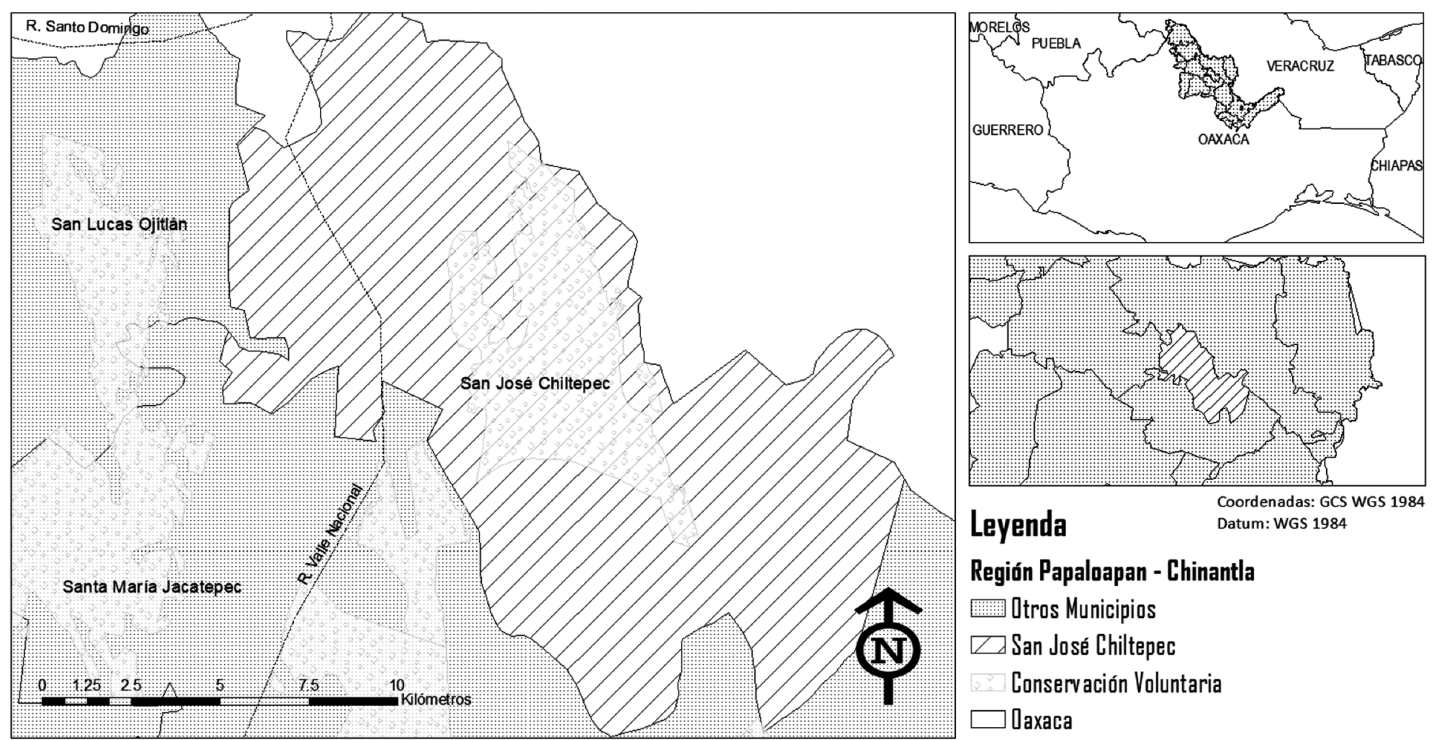

Figura 1. Ubicación Geográfica del área de estudio y polígono de área destinada voluntariamente para la conservación.

drenaje; $17.1 \%$ no dispone de agua entubada de la red pública; y $8 \%$ no tiene excusado o sanitario. Destacando por la carencia de servicios las comunidades de Arroyo Choápam, Cerro Flores, San Isidro Naranjal, Plan de Águila y Pueblo Viejo (CONEVAL, 2010). De acuerdo a BIOGEMATICA (2012) la mayoría de la población se abastece de afluentes del Río Valle Nacional y de pozos que están bajo control sanitario; y el servicio de drenaje está identificado como fosas sépticas, pero carece de sistemas de tratamiento.

\section{Análisis del proceso de establecimiento del Comité Comunitario}

Se convocó a los diferentes actores locales a talleres participativos a través de la asamblea comunitaria para la mejora de las condiciones ambientales e hidrológicas de San José Chiltepec como lo señala (Adhikari et al., 2014). Se realizó una investigación documental sobre la información del municipio y el ejido con apoyo de las autoridades municipales y ejidales para incluir los diagnósticos territoriales en el diseño de herramientas metodológicas a utilizar.

Los talleres se diseñaron bajo una secuencia lógica de capacitación-resultado:

1) Sensibilización respecto a la importancia del tema hídrico (Adhikari et al., 2014), incluyendo seis temas para establecer una línea de conocimiento base y lenguaje común: a) derecho al agua y el saneamiento como derecho humano; b) análisis rápido de los principales contaminantes de los cuerpos de agua; c) características de los cuerpos de agua; d) situación del agua en México; e) reglamentos y normativas para el uso y manejo del agua; y f) iniciativas por el agua; bajo una dinámica expositiva.

2) Identificación de problemática comunitaria a atender mediante cuatro preguntas detonadoras: a) ¿Cuál es la problemática a atender?; b) ¿Cómo la podemos atender?; y c) ¿Quiénes deben atenderla?; utilizando una dinámica de integración y trabajo en equipos (Caron, 1996; Fetterman, 2005).

3) Integración de objetivos para el establecimiento de un comité comunitario basados en los planteamientos Perevochtchikova y ArellanoMonterrosas, (2008) y el enfoque metodológico teórico - práctico hacia el empoderamiento (Fetterman, 2005).

En todos los talleres participativos se incluyeron tres principios o formas de actuar (Caron, 1996): a) escuchar y comentar para decidir mejor; b) trabajar en equipo para reconocer el esfuerzo de los demás y reconocer su actuar como aportación y; c) reflexionar de forma conjunta la problemática para construir soluciones en base a objetivos 
comunes y compromisos mutuos. La información se documentó a través de relatorías y en cada uno de los talleres material fotográfico.

\section{Resultados}

En el taller de sensibilización sobre el tema hídrico acudieron 16 personas, incluidas las autoridades ejidales y municipales, representantes del sistema de la radio comunitaria, líderes locales e interesados en la mejora de su comunidad; quienes definieron su postura mediante seis pronunciamientos base: a) identificación, ubicación, cuantificación y periodicidad de sus recursos hidrológicos. "No se puede defender y proteger lo que no se conoce"; b) el derecho universal del acceso a agua limpia de calidad y suficiencia para las actuales y nuevas generaciones; $\mathrm{c}$ ) involucramiento de la población en esta importante tarea, con énfasis en sumar a los jóvenes/futuros dueños del territorio; d) establecer mecanismos de control, cuidado y protección de sus principales fuentes de agua; e) asegurar la soberanía de la comunidad a sus fuentes de agua; f) diseño e implementación de eventos de capacitación continuos.

Al taller orientado para la identificación de la problemática a atender, acudieron 24 personas incluidas aquellas que participaron en el primer evento. La problemática identificada se puede resumir en que: a) la mayoría de los cuerpos de agua se están degradando por contaminación; b) se desconoce en términos generales el número de cuerpos de agua -permanentes o intermitentes- existentes en la comunidad, se sabe a nivel personal su existencia; c) no existe conocimiento respecto al estatus legal de los cuerpos de agua, es decir, se sabe quién los posee pero no de quien son; y d) no se conoce los medios legales para asegurar su protección, su pertenencia y su disfrute. Se plantearon tres propuestas de solución: a) la creación de un comité comunitario para la protección y manejo del agua; b) el diseño de un reglamento interno que considere la revisión de leyes y reglamentos en la materia; c) la realización de talleres y capacitación direccionado a todos los sectores y actores de la comunidad para este importante esfuerzo.

En el taller para definir objetivos para el establecimiento de un comité comunitario, acudieron el mismo número de personas y se mejoraron los pronunciamientos del primer evento para tener claridad sobre los elementos que regirían al comité para el manejo del agua (Tabla 1). Considerando, que el documento a elaborar tendría que ser coadyuvante a los esfuerzos y a las iniciativas emprendidas por el ejido en materia de conservación y manejo del recurso agua, como un recurso natural y al que el acceso a la población debe ser garantizado como un derecho universal sin distinción de raza, credo, preferencia sexual, sexo, edad o cualquier otro motivo racista que implique discriminación.

Se planteó que se estableciera a nivel ejidal un Comité Comunitario para el Manejo del Agua que funcionará de manera colegiada y que las decisiones que se tomaran, fueran por mayoría de

Tabla 1. Principios fundamentales del comité comunitario de San José Chiltepec resultado del taller definitorio de objetivos locales.

1. El agua es un servicio ambiental, no creado por el hombre y derivado del cuidado y manejo de bosques y selvas. No reconoce fronteras geográficas ni administrativas o políticas, tampoco diferencia expresiones culturales o religiosas, y en tal sentido es una obligatoriedad universal su cuidado y manejo.

2. Se rechaza cualquier iniciativa, posicionamiento, acción o esfuerzo privado o gubernamental que tenga por motivo la privatización o la incorporación del recurso hídrico a la economía de mercado y en especial cuando tenga como fin el uso para el aprovechamiento en la minería o industrias energéticas.

3. El desarrollo de marcos legales e institucionales a nivel local, debe ser consensuado con los ejidos, comunidades y pueblos mediante consultas públicas que garanticen el acceso a la información libre, previa e informada, en especial en un marco de respeto a los usos y costumbres vigilando el cumplimiento de las salvaguardas sociales.

4. El recurso hídrico es un bien común, universal y estratégico para el desarrollo de los pueblos y naciones, por consecuencia los esfuerzos para la conservación y manejo del agua deben ser compartidos al igual que los costos incrementales que de esto se derivan.

5. Es una obligatoriedad de todo ser humano emprender acciones y medidas que tengan por objeto, el cuidado, el buen manejo y la distribución justa y equitativa del recurso hídrico, para asegurar igualdad de oportunidades para el desarrollo humano, social y cultural. 
votos. Se definieron funciones de representatividad basadas en la asamblea, que fuera coordinada con los integrantes del comisariado ejidal y del consejo de vigilancia para poder accionar procesos de gestión y ejecución de proyectos en materia de recursos hidrológicos y otras encomiendas que en materia de agua fueran encomendadas por la asamblea.

Se estableció una periodicidad para el sesionar del cuerpo representativo de al menos una vez al mes para revisar de manera puntual los asuntos que fueran planteados por los integrantes del comité comunitario y el levantamiento de minutas de trabajo para evaluar el cumplimiento de los compromisos establecidos. Se planteó la elaboración de un programa de trabajo anual, que tendría que ser validado por la asamblea ejidal y enriquecido por los integrantes del ejido. Para su operatividad se planteó que funcionaran con los recursos financieros que gestionaran o que se le designaran mediante la asamblea o aquellos que le fueran aportados por autoridades municipales, estatales, federales; o bien donativos públicos o privados, pero que tuvieran su origen en actividades lícitas o sustentables. Los integrantes del comité comunitario del agua, durarían en su cargo tres años y serían electos de conformidad con los lineamientos que estableciera la asamblea y que podría existir reelección por una sola ocasión. Á su vez que el Comité Comunitario para el Manejo del Agua, podría coordinarse con otros comités que se establecieran en la asamblea, cuerpos técnicos municipales, estatales, federales, o con organismos de la sociedad civil y representantes gubernamentales para complementar recursos y hacer más eficiente su actuar.

\section{Discusión}

El informe sobre los Objetivos del Milenio de la Organización de las Naciones Unidas, señala que deberían usarse las experiencias disponibles para iniciar procesos de actualización y rediseño de políticas públicas, garantizando el acceso al agua en cantidad suficiente y de buena calidad para el consumo humano, las actividades productivas y el mantenimiento de los servicios ecosistémicos, y sobre todo para sanear el agua existente, ayudando a recuperar el volumen de los acuíferos (Carrasco y Vargas, 2012).

En este sentido la investigación consideró la aproximación participativa para la mejora de condiciones ecológicas a través de una normativa comunitaria, la cual ha demostrado éxitos (Adhikari et al., 2014; Yang, 2015); el enfoque de participación comunitaria (Tsouvalis y Waterton, 2012) para acercarnos a un modelo de Gobernabilidad Democrática del Agua (Ochoa, et al., 2011) mejor conocido como Organización Comunitaria de Servicios de Agua y Saneamiento (OCSAS); que funcionan bajo bases fundamentales y aspectos complementarios (Tabla 2); muy similares a los que resultaron del taller para definir objetivos para el establecimiento del comité comunitario (Tabla 1); y elementos planteados por el principio de diseño de los comunes (Ostrom, 2015) para el acercamiento e identificación de las prioridades y actores locales; lo que permitió avanzar en el proceso de sensibilización, vinculación y afianzamiento de la lógica de acción colectiva para la transición de la planeación a la acción sobre los recursos hidrológicos.

En este sentido, en México son pocos los casos documentados donde se da el manejo

Tabla 2. Bases del modelo de Gobernanza Democrática del Agua

\begin{tabular}{ll}
\hline \multicolumn{1}{c}{ Bases fundamentales del modelo } & \multicolumn{1}{c}{ Aspectos Complementarios } \\
\hline $\begin{array}{l}\text { Privilegie el acceso al agua para consumo humano } \\
\begin{array}{l}\text { Incluya la participación ciudadana en la toma de decisiones, } \\
\text { de manera que la gobernabilidad sea democrática, legítima y } \\
\text { representativa }\end{array}\end{array}$ & $\begin{array}{l}\text { Respete la diversidad e interpretación cultural del "valor" del } \\
\text { agua (perspectiva de interculturalidad) }\end{array}$ \\
\hline Cumpla con las condiciones de sustentabilidad & $\begin{array}{l}\text { Incorpore todas las alternativas de vinculación entre los distintos } \\
\text { sectores sociales, empresariales y públicos }\end{array}$ \\
\hline $\begin{array}{l}\text { Pueda ser replicable, adaptándose según el contexto local/ } \\
\text { nacional }\end{array}$ & $\begin{array}{l}\text { Favorezca iniciativas de incidencia en políticas para promover } \\
\text { la Gobernanza Democrática del Agua }\end{array}$ \\
\hline
\end{tabular}

Fuente: En (Ochoa, et al. 2011). 
comunitario del agua (Galindo y Palerm, 2016; Jiménez, 2016; Perevochtchikova et al., 2016; Cervantes et al., 2017; Sánchez y Alvarado, 2017) si consideramos que hay más de 80.000 OCSAS en Latinoamérica (Ochoa, et al., 2011); todos ellos trabajando de forma voluntaria y sin remuneración alguna, desempeñando su función por vocación o compromiso social, pero que ha establecido mecanismos de inicio democráticos (Jiménez, 2016) que son validados a nivel comunitario y que buscan garantizar el acceso sostenible a servicios de agua y saneamiento, situación presente en el comité comunitario de San José Chiltepec (Cervantes et al., 2017).

La gestión del territorio a través de las cuencas hidrológicas y microcuencas que no excluyan, ignoren y confronten a los actores locales (Nepal, 2002; Yang et al., 2015), no es tarea simple y el proceso de adaptación para la implementación de un comité comunitario, aunque en la teoría resulte idónea es compleja. Los resultados del taller de sensibilización comunitaria, responden al planteamiento de Perevochtchikova y Arellano-Monterrosas (2008) sobre la elaboración de planes de desarrollo para la gestión del agua basado en la percepción local mediante comités de manejo comunitario. A su vez, la inclusión de los múltiples actores locales que inciden en el territorio (Karelakis et al., 2013) para realizar el consenso de planes y mejoras, son elementos comunes dentro del proceso de formación de las OCSAS (Ochoa, et al., 2011) al ser grupos de vecinos o avecindados donde no llega el agua, es deficiente el servicio o enfrentan serios problemas sanitarios, tal como sucede en San José Chiltepec.

\section{Conclusión}

La mayoría de estudios de casos exitosos de comités comunitarios para el manejo del agua, están basados en mecanismos en operación existentes y no en la creación o implementación de uno nuevo, por lo que no existe una receta o manual que garantice la implementación perfecta o el manejo exitoso del recurso hídrico por parte de estos comités comunitarios.

El caso del ejido de San José Chiltepec resulta destacable puesto que la creación de un reglamento mediante la toma de consciencia hacia un cambio positivo en el actuar y coaccionar de sus habitantes, en la solución de los problemas hidrológicos no es tarea fácil. Sin embargo, el respaldar las acciones a impulsar por los diferentes actores locales y lograr una validación ante la asamblea comunitaria en el caso de ejidos es ya un éxito en sí mismo.

Este proceso de participación y gestión comunitaria a través del trabajo voluntario de los actores locales ha sumado esfuerzos para lograr extender los beneficios a las comunidades cercanas, quienes tienen arraigo hacia el cuidado de su entorno por la cosmovisión idígena existente en la zona. Es necesario impulsar el fortalecimiento de capacidades del comité comunitario para la toma de decisiones; situación que tendrá que realizarse en la medida que existan las condiciones y la voluntad de atender a los procesos, requiriendo de mayor tiempo y un acompañamiento constante para que puedan operar de forma independiente $\mathrm{e}$ impulsar la mejora de actividades agropecuarias para optimizar el uso del agua; un programa de monitoreo sobre la calidad del agua; y el posible establecimiento de cuotas y sanciones a nivel comunitario para la operación correcta del sistema.

\section{Agradecimientos}

Los autores expresan su agradecimiento a por las facilidades brindadas para realizar este estudio a EcoLogic Development Fund, Fondo Ambiental Regional de la Chinantla Oaxaca, A.C., y el ejido de San José Chiltepec. Así como a los comentarios de Leonardo Hernández por sus comentarios durante la revisión de este artículo.

\section{Literatura Citada}

Adhikari, S.; Kingi, T.; Ganesh, S.

2014. Incentives for community participation in the governance and management of common property resources: the case of community forest management in Nepal. Forest Policy and Economics, 44: 1-9.

Ballesteros, B.J.; Murillo, J.M.

2010. Actuaciones de Uso Conjunto Realizadas en la Provincia de Alicante: La Marina Alta. En: Murillo,
J.M..; López, J.A..; Rodríguez, L. Desarrollo Sostenible, Uso Conjunto y Gestión Integral de Recursos Hídricos. Instituto Geológico y Minero de España. Alicante. España. Pp. 210-379.

Biogemática

2012. Atlas de Riesgos (o Peligros) Naturales del Municipio de San José Chiltepec 2011. SEDESOL. San José Chiltepec, México. 115 p. 
Caron, J.

1996. Una teoría ecológica para la intervención comunitaria: acceso y conservación de los recursos. Intervención Psicosocial. Colegio Oficial de Psicólogos de Madrid, 5 (14): 53-68.

Carrasco, G.B.; Vargas, J.J.

2012. Manejo del agua desde las agendas de los organismos internacionales y sus interpretaciones locales en México. Políticas públicas para el manejo de la cuenca LermaChapala, en su porción ubicada en el estado de México. Quivera, 14: 66-77.

Cervantes, A.; Velázquez, M.; Pimentel, J.L.

2017. Gobierno y administración local del agua potable en la Ciénega de Chapala, Michoacán, México. Tecnología y Ciencias del Agua, 8 (1): 65-80.

CONAGUA

2016. Estadísticas del Agua en México. Edición 2016. SEMARNAT. México DF., México 275 p.

\section{CONEVAL}

2010. Informe anual sobre la situación de pobreza y rezago social. Edición 2010. SEDESOL. San José Chiltepec, Oaxaca. México. 2 p.

DeFries, R.; Hansen, A.; Newton, A.C.; Hansen, M.C.

2005. Increasing isolation of protected areas in tropical forests over the past twenty years. Ecological Applications, 15 (1): 19-26.

Dourejani, A.; Andrei, J.

1999. Gestión de cuencas y ríos vinculados con centros urbanos. Comisión Económica para America Latina y el Caribe. CEPAL, Santiago, Chile. 176 p.

Fetterman, D.M.

2005. Empowerment Evaluation: From the Digital Divide to Academic Distress. En: (Fetterman, D.M.; Wandersman, A.). Empowerment Evaluation Principles in Practice. Guildford Press. New York. US. 92-122.

Galindo, E.; Palerm, J.

2016. Sistemas de agua potable rurales. Instituciones, organizaciones, gobierno, administración y legitimidad. Tecnología y ciencias del agua, 7 (2): 17-34.

Jiménez, A.A.

2016. La participación social y la gestión del agua en comunidades rurales de Jalisco. Económica Regional, 14 (80): 43-49.

Liu, J.; Ouyang, Z.; Miao, H.

2010. Environmental attitudes of stakeholders and their perceptions regarding protected area-community conflicts: a case study in China. Journal of Environmental Management, 91 (11): 2254-2262.

Karelakis, C.; Zafeiriou, E.; Galanopoulos, K.; Koutroumanidis, K. 2013. Natural Resources in Regional and Rural Development: Moving from Public Opinions to Policy Action. New Medit, 13: 56-64.

Nepal, S.K.

002. Involving indigenous peoples in protected area management: comparative perspectives from Nepal, Thailand, and China. Environmental Management, 30 (6): 748-63.

Ochoa, E.; Soto, L.; Burt, P.

2011. Organizaciones Comunitarias de Servicios de Agua y Saneamiento. En: Vagliente, P.. Modelos de Gobernabilidad Democrática para el Acceso al Agua en América Latina. Fundación AVINA. Argentina. 21-52.

OECD

2013. OECD Evaluaciones de la OCDE sobre el desempeño ambiental: México 2013, OECD Publishing. Mexico. Pp. 29-35.

Ostrom, E.

2015. Governing the commons. Ed. 2015. Cambridge University Press. Cambridge. United Kingdom. 294 p.

Perevochtchikova, M.; Arellano-Monterrosas, J.L.

2008. Gestión de cuencas hidrográficas: experiencias y desafíos en México y Rusia. Revista Latinoamericana de Recursos Naturales, 4 (3): 313-325.

Perevochtchikova, M.; Aponte Hernández, N.; Zamudio-Santos, V.; Sandoval-Romero, G.E.

2016. Monitoreo comunitario participativo de la calidad del agua: caso Ajusco, México. Tecnología y Ciencias del Agua, 7 (6): 5-23.

Sánchez, G.J.; Alvarado, E.P.

2017. Redes de gobernanza y organizaciones que intervienen en la gestión pública de agua en la esfera local: comités de agua potable del municipio de Xalatlaco, México. Journal de Ciencias Sociales, 5 (8): 65-87.

Tsouvalis, J.; Waterton, C.

2012. Building "participation" upon critique: The Loweswater Care Project, Cumbria, UK. Environmental Modelling \& Software, 36: 111-121.

Yang, H.; Harrison, R.; Yi, Z.F.; Goodale, E.; Zhao, M.X.; Xu, J.C. 2015. Changing perceptions of forest value and attitudes toward management of a recently established nature reserve: A case study in southwest China. Forests, 6 (9): 3136-3164. 
